\title{
Lumbo Sacral Fixation After Lumbosacral Decompression for Disc and Spondilotic Changes Along with Instability and Stabilization with MRI Compatible Titanium Pedical Screw and Rods Fixation Under Neurophysiological Monitoring to Reduce Morbidity and Complication, An Experience of 30 Years
}

\author{
PK Upadhyay ${ }^{*}$, G Tiwary ${ }^{2}$, K Upadhyay ${ }^{3}$ and Kritika $\mathbf{U}^{3}$ \\ ${ }^{1}$ Head of Neurosurgery Department, Institute of Human Behavior and Allied Sciences \\ Hospital, Dilshad Garden, Delhi, India \\ ${ }^{2}$ Director Laboratory Sciences, House of Diagnostic, Delhi, India \\ ${ }^{3} \mathrm{MBBS}$ Fellow (JSSMC and MAMC), India
}

Received: December 20, 2021

Published: January 07, 2022

(C) All rights are reserved by PK Upadhyay.,

et al.

*Corresponding Author: PK Upadhyay, Head of Neurosurgery Department, Institute of Human Behavior and Allied Sciences Hospital, Dilshad Garden, Delhi, India.

\begin{abstract}
130 cases operated by the senior author in 30 years utilizing neurophysiological monitoring, using lumbosacral discectomy and decompression for canal stenosis and destabilization by immediate fixation using MRI compatible titanium pedicle rods and screw under intraoperative Neurophysiological monitoring of somato sensory evoked potential and motor evoked potential, which helped immensely in preventing damage to neural structure and decreasing the chances of mortality and morbidity for patients and confidence and reliable method for neuro-spinal surgeon.
\end{abstract}

Keywords: Lombo Sacral; Pedicle Screws and Rods; MRI Compatible; Titanium Screw and Rods; Intraoperative Neurophysiological Monitoring; SSEP, MEP; SEP;

\section{Introduction}

All weight and pressure put on posterior element of the spine are ultimately passes through the pedicles to the vertebral body. The pedicle screw placed to the body involves all three columns described by Denis in spinal instability. In 1982 A D Steffee found that that the pedicles are the strongest part of the spine to fix from posterior to anterior and developed variable screw placement (VSP) systems [1,2].

All movement are well restricted by pedicle screw fixation. It has advantage of providing immediate rigid segmental fixation, avoid the spinal canal and thus can be utilized even when laminectomy has been done. It can also be used when bone is osteoporotic.
It helps in early mobilization post operatively and no bracing is required and decreases hospitalization days and decreased level of nursing care as well $[3,4]$.

The limitation was that it was not MRI compatible and used to give a lot of artifacts on CT scanning so post operative evaluation was difficult or not possible.

MRI compatible titanium screw and plate overcame this disadvantage and it was now possible to do ost operative evaluation if need arise be in future. Use of Neurophysiological monitoring also decreased chances of injury to neural structure during surgery or screw placement and in many case almost eliminated the neural damage together. 
Lumbo Sacral Fixation After Lumbosacral Decompression for Disc and Spondilotic Changes Along with Instability and Stabilization with MRI Compatible Titanium Pedical Screw and Rods Fixation Under Neurophysiological Monitoring to Reduce Morbidity and Complication, An Experience of 30 Years

\section{Materials and Methods}

130 cases operated by the senior author in 30 years utilizing neurophysiological monitoring. out of which 80 cases were males and 50 cases were females. 55 cases were in age group of 30 to 40 years. 47 cases were in age group of 50 to 60 years and years 15 case in 20 to 30 years and 13 cases in 70 to 80 years.

The procedure is utilized where there is instability following fracture dislocation or due to lumber spondylosis. It was utilized in cases were instability was likely to develop immediately after operation or there was chances of development of delayed instability or deformity, after primary surgery like lumbosacral discectomy and decompression for canal stenosis or reduction of fracture dis- location and stabilization by immediate fixation using MRI compatible titanium pedicle rods and screw under Neurophysiological monitoring of somato sensory evoked potential and motor evoked potential, which helped immensely in preventing damage to neural structure and improving results, both for spinal surgeon and the patients. The Neurophysiological monitoring was done intraoperatively only to prevent intraoperative neural damage.

Since the neurophysiological monitoring was done in only intraoperative phase therefore pre operative and post operative comparison is not possible.

Pre operative pictures of illustrative patients are shown in table 1.

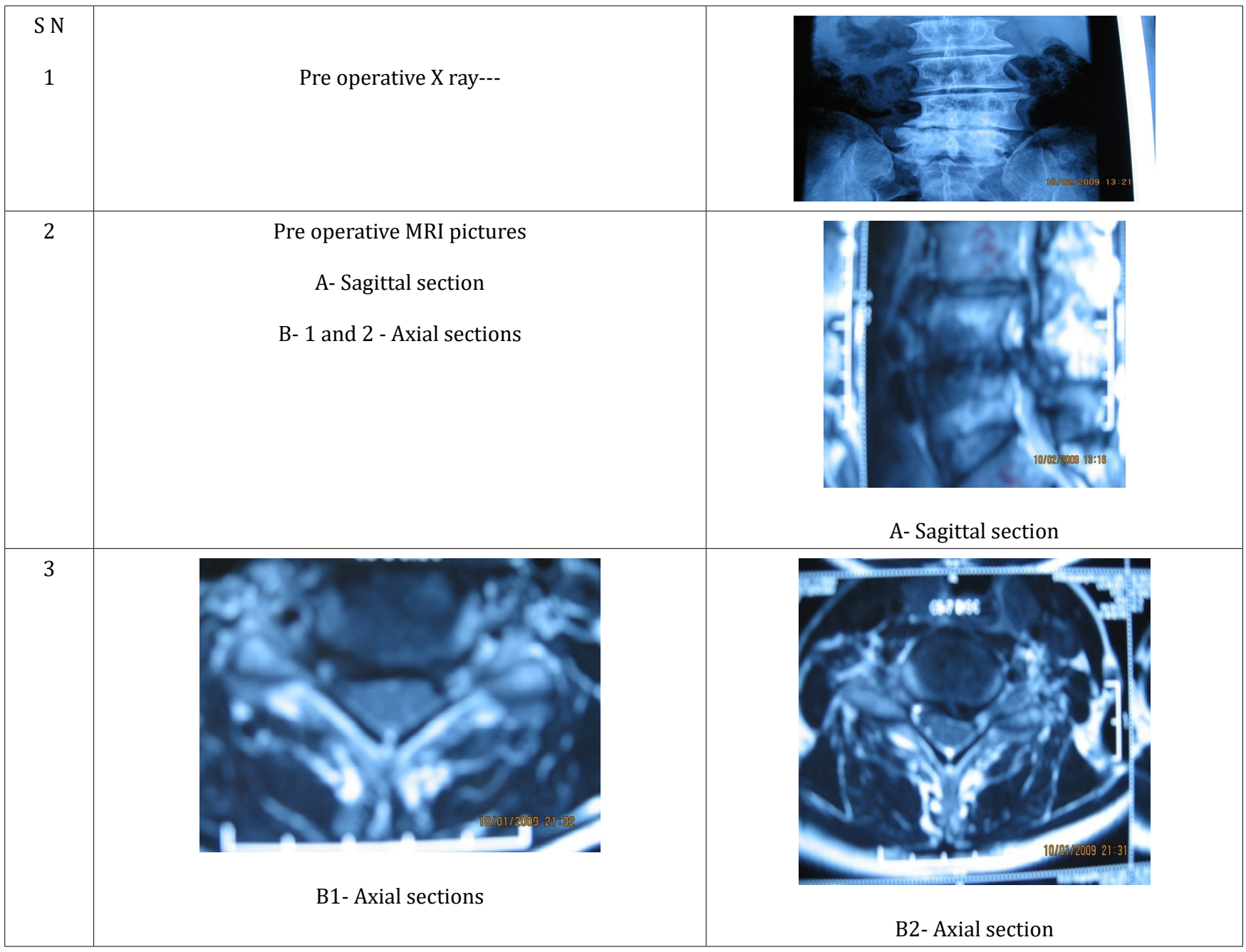

Table 1: Pre operative X- Rays and MRI photographs. 
Lumbo Sacral Fixation After Lumbosacral Decompression for Disc and Spondilotic Changes Along with Instability and Stabilization with MRI Compatible Titanium Pedical Screw and Rods Fixation Under Neurophysiological Monitoring to Reduce Morbidity and Complication, An Experience of 30 Years

Operative procedures through standard posterior spinal route to the lumbar spine were used. Spine, lamina, transverse process and auricular joints are exposed at the concerned level and one level up and one level down.

Screw entry point and pedicle centre. We follow the standard Steffee procedure for the pedicle screw placement. In lumber region the convergence of the ridge on the superior articular facet, the ridge on the pars interarticularis and ridge on the transverse process, represents the screw entry point $[1,3]$.

Radiological guidance using image intensifier or digital intra operative $\mathrm{x}$-ray may be used to guide the screw position to the pedicle. Ones crews are placed bilaterally the plate is attached and fixed with other screws over the plates. Extra large portion of screw then may be cut after tightening the nuts.

Now we do rod placement in place of plates. Advantages are now they are available in titanium so they are MRI compatible and re evaluation may be done in future id need arise be. It also has advantageous as rods are long and can be placed at any length while plates were of fixed size or even if variable plate are used there was very less variation in length possible.

After placement haemostasis is done and muscles are closed in three layers as standard practice at our institution and then subcutaneous and skin is closed and dressing is done after cleaning with saline and spirit and antibiotic iodine lotion. Antibiotic coverage is given till suture is removed.

Post operative complications encountered were 05 superficial wound infections.

Intraoperative X- Rays and intra operative photo with post operative photo of patient shown in table 2 .

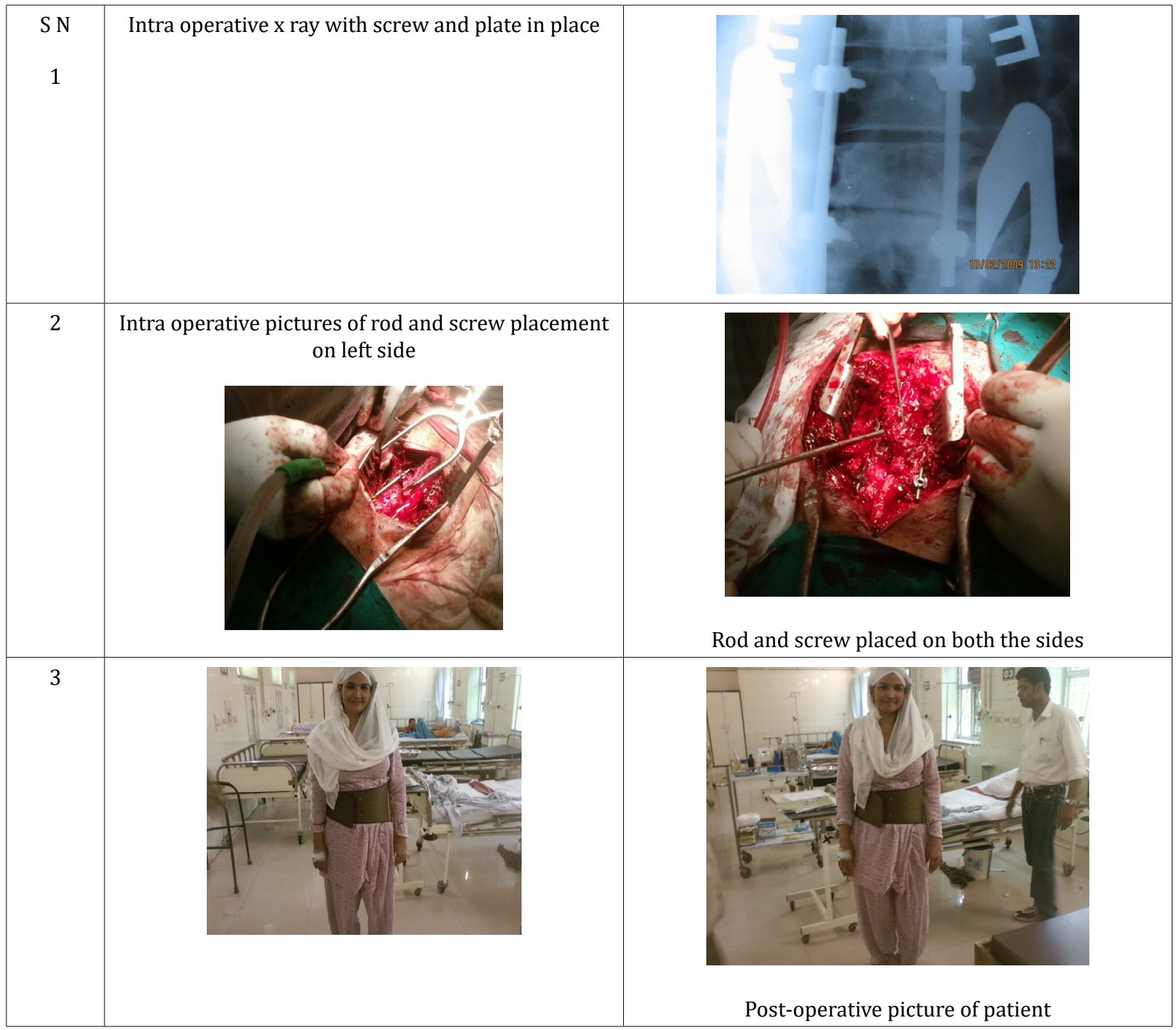

Table 2: Intra operative x-rays and phot graph of the screw and rod placement and post operative picture of patient at serial no 3. 
Lumbo Sacral Fixation After Lumbosacral Decompression for Disc and Spondilotic Changes Along with Instability and Stabilization with MRI Compatible Titanium Pedical Screw and Rods Fixation Under Neurophysiological Monitoring to Reduce Morbidity and Complication, An Experience of 30 Years

Two displacement of rods due to non compliance of patient who scatted against advise. These require replacement using bone cement in the pedicle perforations. No increase in Neurological deficit was encounterd in any of the cases operated under intraoperative Neurophysiological monitoring incomparision to preoperative status.

\section{Neurophysiological monitoring (IONPM)}

Intraoperative neurophysiological monitoring (IOM) in spinal surgery reduces the incidence of postoperative neurological complications, at all neurological level. Many techniques are available and motor and somatosensory evoked potentials are thought to be essential for better results of IONPM. Spinal cord evoked potentials (SEP) are observed and recorded over cord. Electrical stimulation is given on the dorsal of spinal cord by an epidural electrode. Somatosensory evoked potentials (SSEP) provide the functional continuity of sensory pathways. That's so from the terminal nerve through the dorsal column going up till the sensory cortex.

Motor evoked potentials (MEPs) consist of spinal, neural and muscular MEPs. MEPs perform selective and specific examination of the working intactness of descending motor pathways, starting from the motor cortex to the terminal muscles. Neurological surgeons must understand the monitoring techniques and read monitoring records properly to understand use IONPM for the proper decision making during the neurosurgery for safe spinal neurosurgery and better surgical results.

\section{Somatosensory evoked potentials (SSEP)}

SEPs were first utilized in the 1970s to assess and monitor the spinal cord function during spinal-surgery for scoliosis treatment. After stimulating terminal nerves, SEPs are measured both from the spinal cord, epidural electrode and/or simultaneously from the sensory cortex of the brain [6]. Normally, the posterior tibial nerve is used for taking ssep traces.

Used data are stimulation, $0.2 \mathrm{~ms}$ duration; at, $-3 \mathrm{~Hz}$ frequency; with intensity $-25 \mathrm{~mA}$. This is given for one minute and averaging gives SSEP result [7]. Latency and amplitude are measured and determined. Latency measures the time and measures distance. Amplitude measures the power and is more variable vis a vis latency. Monitoring of dorsal column intactness by SSEP is the commonest form in neurospinal surgery.

Subdermal needle electrodes, used are made of Platinum. It is used for stimulation and recording. Normally, following is considered.
$50 \%$ decrease in amplitude (b) along with 10\% increase in latency in comparison to baseline values of the patients, makes a warning signal. False negative SSEP monitoring happening during spinal surgery in $0.063 \%$ [4]. Multicenter, very vast, research has concluded the result in reduction of postoperative paraplegia by more than 50\%-60\% with modality [5]. SSEP signals are good indicators of spinal cord function. But much better information about function of nerve root are provided by use of motor evoked potentials.

Direct waves MEP (or spinal motor evoked potentials) these waves are compound corticospinal action potentials started by the direct activation of axons and velocity (conduction) of nearly $50 \mathrm{~m} / \mathrm{s}$ [6], thus making it useful for monitoring the motor pathways up from the motor cortex up to level of the spinal electrode placement. This is gained by single transcranial electrical stimulation of the intensity by, $80-100 \mathrm{~mA}$, and the total stimulus duration of 0.5-1 ms, using normal frequency of 0.5-2 Hz. Recording of which done from the epidural/subdural space of cord [7]. This is directly generated electrical pulse. And thus called "single stimulus technique" of MEP or "spinal motor evoked potentials (MEPs)". This does not require an averaging, but if few averages are taken it improves quality of MEP. This is also good because it provides real-time reading clinically. Warning sign are the decrease in wave amplitude by or more than 50 percent of original baseline value. Or when they cannot be detectable. This may indicate happening of or high probability of severe neurological deficits which may include injuries such as permanent paraplegia.

Other measurements can be used

Neurogenic MEP

- $\quad$ Muscle MEP (or myogenic MEP)

- $\quad$ Spontaneous electromyography

Spontaneous or free-running electromyography (EMG) is used to see or observe selective nerve root functioning undergoing neurospinal- cord spinal surgery. SEP and SSEP data are not real time. But EMG is truely "real-time" data observed from terminal muscles. Spontaneous EMG thus may iliminate operative radiculopathy while spinal instrumentation procedure is being done. This also may include pedicle screw putting. Here no stimulation is required. This can be recorded continuously from particular peripheral muscle or muscle groups supplied by particular nerve roots which are at risk during operation [8-14]. 
Lumbo Sacral Fixation After Lumbosacral Decompression for Disc and Spondilotic Changes Along with Instability and Stabilization with MRI Compatible Titanium Pedical Screw and Rods Fixation Under Neurophysiological Monitoring to Reduce Morbidity and Complication, An

Experience of 30 Years

Method of triggered EMG for observation of the intactness of lumbar pedicles while doing screw placement surgery and the accuracy screw putting was described in 1922 by Calancie., et al. [15].

There is a decrease in electric threshold leading to immediate appearance of CMAPs of the muscles under consideration by the irritated or damaged nerve root that muscle group due to stimulation using the screw [16].

\section{Spinal cord evoked potentials}

SCEP technique was first described in Japan during 1970s. Electrical stimulation was put on the dorsal spinal cord by epidural electrodes during procedure [17] and evoked potentials are recorded over the spinal cord. The SCEP correspond to total of neural activities that originating from the upgoing and down coming tracts and neurons at the site of recording. The potentials so recorded are quite vigorous. They in reality shows all activity of the tracts of the spinal cord, including dorsal columns and the corticospinal tracts along with others [18]. Therefore practically, SCEP cannot provide accurate inputs regarding motor activities. It's so because of presence of sensory-related potentials as well. These sensory potentials, are large in amplitude, and mask motor potentials. Together with SSEP and MEP, electromyography (sEMG and tEMG) should be a necessary part of multimodality protocol for monitoring lumbar spinal nerve roots. Knowledge and experience of the strength and limitations of each modality helps to optimize and further improve the value of IONM (introperative Neuro monitoring) [19].

Neurophysiological intra operative graph have been shown as graph 1 and 2 .

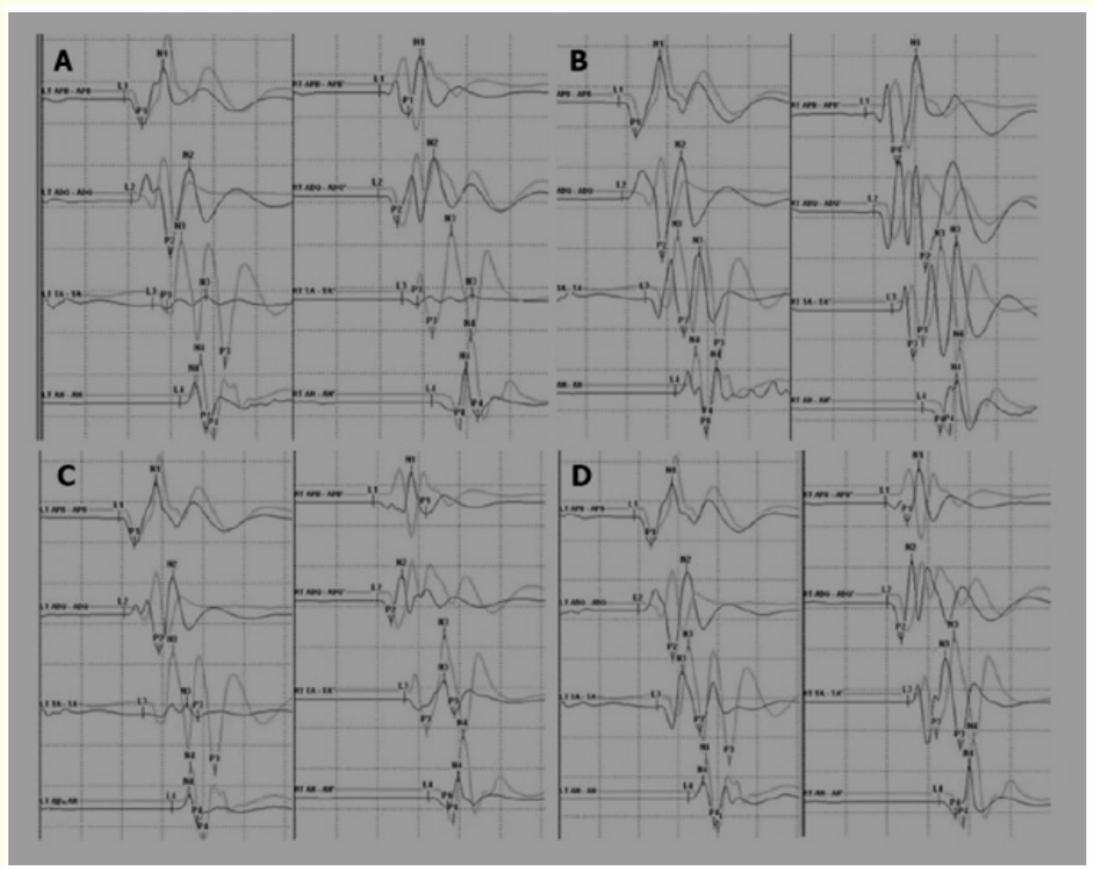

Graph 1: Recording of Neurophysiological monitoring (intra operative).

Representative case demonstrating clinical usefulness of intraoperative neuromonitoring in spinal surgery. A: MEP after applying rod to the screw heads using derotation maneuver and cantilever maneuver. The amplitude of MEP (black line) at both lower more than $50 \%$ compared with the baseline amplitude (green line); B: The amplitude of MEP recovered after correction release by removal of the rods and set screws; C: The amplitude of MEP re-deteriorated extremities decreased after reassembly of the implants; D: The amplitude of MEP recovered finally after raising MAP and administration of dexamethasone. APB: Abductor pollicis brevis; ADQ: Abductor digitiquinti; TA: Tibialis anterior; AH: Abductor halluces; MEP: Motor evoked potential; MAP: Mean arterial pressure. 


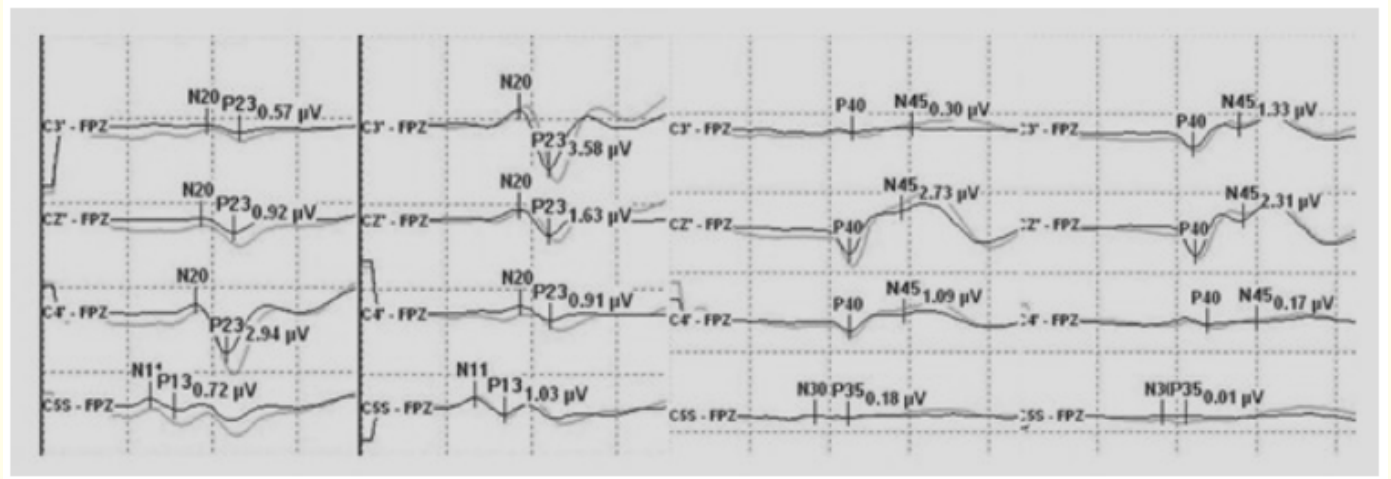

Graph 2: SSEP showing no change in comparison to base line.

\section{Results and Conclusion}

Total of 130 cases operated and studied in duration of thirty years utilizing intra operative neurophysiological monitoring. The indications were lumbosacral discectomy, decompression for canal stenosis and destabilization. This were done by immediate fixation using MRI compatible titanium pedicle rods and screw under intraoperative Neurophysiological monitoring using somato sensory evoked potential and motor evoked potential. This helped immensely in detecting enchroachment of neural structures and thus alerting the neurospinal surgeon and preventing damage to neural structure. By preventing intraoperative neural structure damage, it helps patients and decrease their mortality and morbidity. It also help the neurospinal surgeon during surgery so he is more confident and objectively able to avert damage. Therefore it's the recommendation of the authors that during neuro-spinal surgeries and spinal fixation the methodologies should be utilized if available. It is also preferable to use this preventive measure in extraspinal cord and intra spinal cord surgeries.

\section{Bibliography}

1. Steffee AD. "The variable srew placement system with posterior lumbar interbody fusion". In LIN PM, Gill K (Eds). Lumber inter body fusion Principles and Techniques in Spine Surgery. Aspen publisher INc. 81 (1989).

2. Steffee AD., et al. "Segmental spines with pedicle srew fixation. A new internal fixation device for disorders of the lumbar and thoracolumbar spine". Clinical Orthopaedics and Related Research 203 (1986): 45.
3. Schneck CD. "The anatomical determinants of lumbar spondylosis". In Ramani PS (Ed).Posterior lumbar inter body fusion". Associated Personnel Services Publication 11 (1989).

4. Nuwer MR., et al. "Somatosensory evoked potential spinal cord monitoring reduces neurologic deficits after scoliosis surgery: results of a large multicenter survey". Electroencephalography and Clinical Neurophysiology 96 (1995): 6-11.

5. Nuwer MR. "Spinal cord monitoring". Muscle Nerve 22 (1999): 1620-1630.

6. Macdonald DB., et al. "Intraoperative motor evoked potential monitoring - a position statement by the American Society of Neurophysiological Monitoring". Clinical Neurophysiology 124 (2013): 2291-2316.

7. Kothbauer KF. "Intraoperative neurophysiologic monitoring for intramedullary spinal-cord tumor surgery". Neurophysiology Clinics 37 (2007): 407-414.

8. Owen JH., et al. "Sensitivity and specificity of somatosensory and neurogenic-motor evoked potentials in animals and humans". Spine (Phila Pa 1976) 13 (1988): 1111-1118.

9. Morota N., et al. "The role of motor evoked potentials during surgery for intramedullary spinal cord tumors". Neurosurgery 41.6 (1997): 1327-1336.

10. Kothbauer KF., et al. "Motor-evoked potential monitoring for intramedullary spinal cord tumor surgery: correlation of clinical and neurophysiological data in a series of 100 consecutive procedures". Neurosurgery Focus 4 (1998): e1. 
11. Ulkatan S., et al. "Monitoring of scoliosis surgery with epidurally recorded motor evoked potentials (D wave) revealed false results". Clinical Neurophysiology 117 (2006): 2093-2101.

12. Deiner S. "Highlights of anesthetic considerations for intraoperative neuromonitoring". Seminars in Cardiothoracic and Vascular Anesthesia 14 (2010): 51-53.

13. Lall RR., et al. "Intraoperative neurophysiological monitoring in spine surgery: indications, efficacy, and role of the preoperative checklist". Neurosurgery Focus 33 (2012): E10.

14. Gonzalez AA., et al. "Intraoperative neurophysiological monitoring during spine surgery: a review". Neurosurgery Focus 27 (2009): E6.

15. Calancie B., et al. "Intraoperative evoked EMG monitoring in an animal model. A new technique for evaluating pedicle screw placement". Spine (Phila Pa 1976) 17 (1992): 1229-1235.

16. Bosnjak R and Dolenc VV. "Electrical thresholds for biomechanical response in the ankle to direct stimulation of spinal roots L4, L5, and S1. Implications for intraoperative pedicle screw testing". Spine (Phila Pa 1976) 25 (2000): 703-708.

17. Tamaki T and Kubota S. "History of the development of intraoperative spinal cord monitoring". European Spine Journal 16 (2007): S140-S146.

18. Jong-Hwa Park and Seung-Jae Hyun. "Intraoperative neurophysiological monitoring in spinal surgery". World Journal of Clinical Cases 3.9 (2015): 765-773.

19. Yuguang Chena Baoqing P., et al. "Neurophysiological monitoring of lumbar spinal nerve roots: A case report of postoperative deficit and literature review". International Journal of Surgery Case Reports 30 (2017): 218-221.

\section{Assets from publication with us}

- Prompt Acknowledgement after receiving the article

- Thorough Double blinded peer review

- Rapid Publication

- Issue of Publication Certificate

- High visibility of your Published work

Website: www.actascientific.com/

Submit Article: www.actascientific.com/submission.php

Email us: editor@actascientific.com

Contact us: +919182824667

Citation: PK Upadhyay., et al. "Lumbo Sacral Fixation After Lumbosacral Decompression for Disc and Spondilotic Changes Along with Instability and Stabilization with MRI Compatible Titanium Pedical Screw and Rods Fixation Under Neurophysiological Monitoring to Reduce Morbidity and Complication, An Experience of 30 Years". Acta Scientific Medical Sciences 6.2 (2022): 11-17. 